\title{
In silico docking analysis of constituents of Zingiber officinale as antidepressant
}

\author{
Sibi P. Ittiyavirah* and Meera Paul \\ Department of Pharmacology, University College of Pharmacy, M.G University, Cheruvandoor Campus, Ettumanoor, \\ Kottayam 686631, Kerala, India.
}

Accepted 15 May, 2013

\begin{abstract}
Depression is among the leading causes of disability worldwide and its global burden seems to further increase in the future. In the present study, in silico approach was used to assess the use of constituents of Zingiber officinale as potential agents that could act as antidepressant agents. Docking studies of Gingerol and Shogoal were carried out using Argus lab 4.0.1. Analysis of the results of the docking software suggested that Gingerol and Shogoal can act as potent antidepressants. For the binding analysis, $5 \mathrm{HT}_{1 \mathrm{~A}}$ receptor protein was taken for the study as it is considered being a potential target for treatment of depression. The standard drug, imipramine was subjected to docking analysis for comparative study. Further analysis in the wet lab may provide more information regarding antidepressant activity of $Z$. officinale.
\end{abstract}

Key words: Zingiber officinale, Argus lab 4.0.1, docking, depression, $5 \mathrm{HT}_{1 \mathrm{~A}}$ receptor.

\section{INTRODUCTION}

Depression is among the leading causes of disability worldwide and its global burden seems to further increase in the future. The extensive human and economic costs stress the importance of studying various aspects of depression. A number of drugs are available for the treatment of depression, but clinical evaluation of these drugs has shown incidence of relapses, side effects, and drug interactions. This has been the rationale for the development of new antidepressants, which includes herbal drugs (Rajput et al., 2011).

Herbal products are often perceived as safe because they are "natural". Herbal medicine is a major component in all traditional medicine systems and a common element in Siddha, ayurvedic, homeopathic, naturopathic, traditional Chinese medicine, and Native American medicine. Considerable efforts have been directed towards the development of natural products from various plant sources which have antidepressant activity. The serotonin $5-\mathrm{HT}_{1 \mathrm{~A}}$ receptor $\left(5-\mathrm{HT}_{1 \mathrm{~A}} \mathrm{R}\right)$ is a G-protein coupled receptor widely expressed in the central nervous system (CNS), where it is involved in the modulation of mood, emotion and depression, and of different behavioral responses, including thermoregulation, sleep, feeding, aggression, and anxiety. Of all the serotonin receptor subtypes, $5-\mathrm{HT}_{1 \mathrm{~A}} \mathrm{R}$ is the dominant receptor responsible for depression and it is involved in the mechanism of action of several antidepressant drugs (Nievergelt et al., 2010). A number of natural antidepressant compounds have been reported in the medicinal plants and tested for their efficacy in treating depression. Therefore, the present study aims to investigate the antidepressant activities of the compounds from Zingiber officinale against the $5-\mathrm{HT}_{1 \mathrm{~A}}$ receptor protein by using Argus lab 4.0.1.

\section{MATERIALS AND METHODS}

Preparation of protein structure

The sequence of the $5 \mathrm{HT}_{1 \mathrm{~A}}$ receptor protein was retrieved from 
protein data-base of National Center for Biotechnology Information (NCBI) (http://www.ncbi.nlm.nih.gov/) in FASTA format (a text based format where a single letter code is used to represent amino acid sequences), and it was searched against selection of the related homologues of query sequence in Protein Drug Bank (PDB) (http://www.pdb.org). The homology modelling requires sequences of known three dimension (3D) structure and the target having above $35 \%$ of similarity. Basic local alignment search tool (BLASTP) (http://blast.ncbi.nlm.nih.gov/Blast.cgi) was used to identify the most suitable template for homology modelling of $5 \mathrm{HT}_{1 \mathrm{~A}}$ receptor protein. The available structure of protein in the protein database are:

Top ten hits (PDB structures)

\section{Rank PDB Hit}

1. $2 \mathrm{bg} 9 \mathrm{~B}$;

2. $2 \mathrm{bg} 9 \mathrm{~A}$;

3. $2 \mathrm{bg} 9 \mathrm{C}$;

4. $2 \mathrm{bg} 9 \mathrm{C}$;

5. 2bg9B;

6. $2 \mathrm{bg} 9 \mathrm{~A}$;

7. $2 \mathrm{bg} 9 \mathrm{C}$;

8. $2 \mathrm{bg} 9 \mathrm{~A}$;

9. $2 \mathrm{bg} 9 \mathrm{E}$;

10. $2 \mathrm{bg} 9 \mathrm{~A}$

The templates and target sequence were aligned using GenThreader Server (Jones, 1999). After careful examination for the potential alignment errors, the automated comparative protein modelling program I-TASSER was employed to build the model (Zhang, 2008). The statistical verification of the model was evaluated with PROCHECK, a structure verification program which relies on Ramachandran plot (Vaseeharan and Valli, 2011), which determines the quality of the predicted structure by assessing various parameters such as lengths, angles and planarity of the peptide bonds, geometry of the hydrogen bonds, and side chain conformations of protein structures as a function of atomic resolution. 3D ligand site server was used to predict the possible binding sites. The amino acid residues LEU453, PHE454, TYR457 were revealed as the binding sites.

\section{Preparation of ligand structures}

All the compounds used for docking study (gingerol, shogoal and imipramine) were selected from ChemSketch. Chemically intelligent drawing interface freeware developed by Advanced Chemistry Development, Inc., (http://www.acdlabs.com) was used to construct the structure of the ligands. Using draw mode of Chemsketch, the ligands were generated and the three dimensional optimizations were done and then saved in $\mathrm{m}$ ol file (a file format for holding information about the atoms, bonds, connectivity and coordinates of a molecule). Molecular weight, partition coefficient and number of hydrogen bond donors and acceptors for the active principles were noted. Geometry optimizations of the ligands were performed according to the calculation method by Argus Lab 4.0.1 software.

\section{Protein-ligand docking using Argus Lab 4.0.1}

$5 \mathrm{HT}_{1 \mathrm{~A}}$ receptor protein was docked against the four ligands using Argus Lab 4.0.1 (Mark A. Thompson, Planaria Software LLC, Seattle, WA, USA, http://www.arguslab.com) to find the reasonable binding geometries and to explore the protein ligand interactions.
Docking of the protein ligand complex was mainly targeted only onto the predicted active site. Docking simulations were performed by selecting "Argus Dock" as the docking engine. The selected residues of the receptor were defined to be a part of the binding site. A spacing of $0.4 \AA$ between the grid points was used and an exhaustive search was performed by enabling "High precision" option in docking precision menu. "Dock" was chosen as the calculation type, "flexible" for the ligand and the A score was used as the scoring function. At maximum, 150 poses were allowed to be analyzed; binding site box size was set to $\left(20 \times 20 \times 20 \mathrm{~A}^{\circ}\right)$ so as to encompass the entire active site. The $A$ score function with the parameters read from the AScore.prm file was used to calculate the binding energies of the resulted docked structures. All the compounds in the dataset were docked into the active site of $5 \mathrm{HT} 1 \mathrm{~A}$ receptor protein, using the same protocol. After completion of docking, the docked protein (protein-ligand complex) was analyzed. The docking poses saved for each compound were ranked according to their dock score function.

\section{RESULTS AND DISCUSSION}

\section{Query search result using BLASTP}

Utilizing BLAST program in the National Center for Biotechnology Information (NCBI), the sequences based on the query $\left(5 \mathrm{HT}_{1 \mathrm{~A}} \mathrm{R}\right)$ were generated. BLAST program increased the sensitivity of the BLAST search and helped to generate the possible alignment between the $5 \mathrm{HT}_{1 \mathrm{~A}} \mathrm{R}$ query sequences and database sequences. This gave ten corresponding PDB hits and alignments.

\section{Construction of 3-D models}

The 3-D models of the templates were constructed using the I-TASSER web server. The target sequences were first threaded through a representative PDB structure library to select the best possible models from PDB, based on the templates and then it does multiple threading alignments and iterative structural assembly simulations.

\section{Verification and validation of the model by PROCHECK, Ramachandran plot}

Verification of the built model was done to ensure whether the model was programmed correctly and the algorithms were implemented. Validation results determined that the distribution of amino acid residues were at the most favorable region in the Ramachandran plot. This is an indication of the stereo chemical quality of the model taken for the structural analysis, and also validated the target-ligand binding efficacy of the structure. Ramachandran plot displays the main chain torsion angles phi, psi $(\varphi, \Psi)$ (Ramachandran angles) in a protein of known structure (Figures 1 and 2). The Ramachandran plot shows the phi-psi torsion angles for all residues in the structure (except those at the chain termini) which were classified according to its regions in 


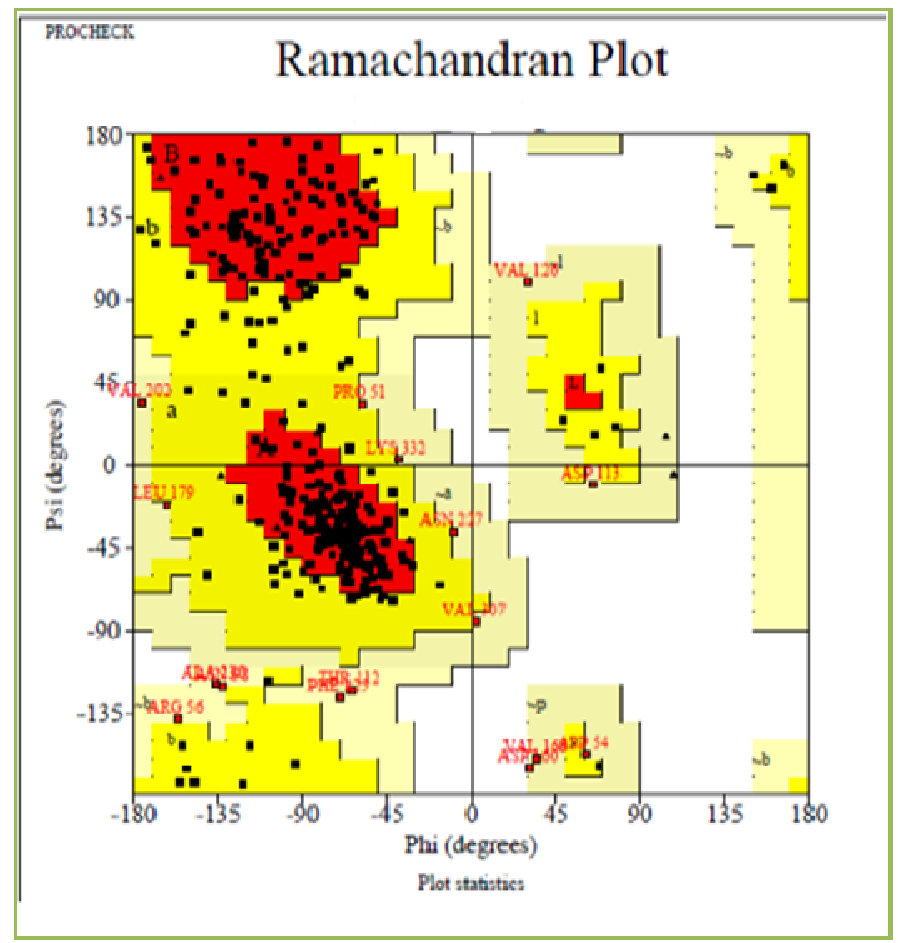

\begin{tabular}{|c|c|}
\hline Residves in most aroved regions $[A, B, L]$ & 248 \\
\hline Residven in additional allow ad region [ablp] & 71 \\
\hline $\begin{array}{l}\text { Kendves un generowly allow ed ragions } \\
{[\sim 2, b, \sim, \sim \phi]}\end{array}$ & 13 \\
\hline Rest ves in dasallow ed regrons & 0 \\
\hline Number of non-glycine and non-proline asidjes & 334 \\
\hline Number of end-residves (excl. Gly and Aro) & 2 \\
\hline Nuaber of lycine ras idve (showa as tiangles) & 2 \\
\hline Number ot poline restdves & 20 \\
\hline Total amber of residves & 67 \\
\hline
\end{tabular}

Figure 1. Ramachandran plot for $5 \mathrm{HT}_{1 \mathrm{~A}}$ protein generated by PROCHECK.

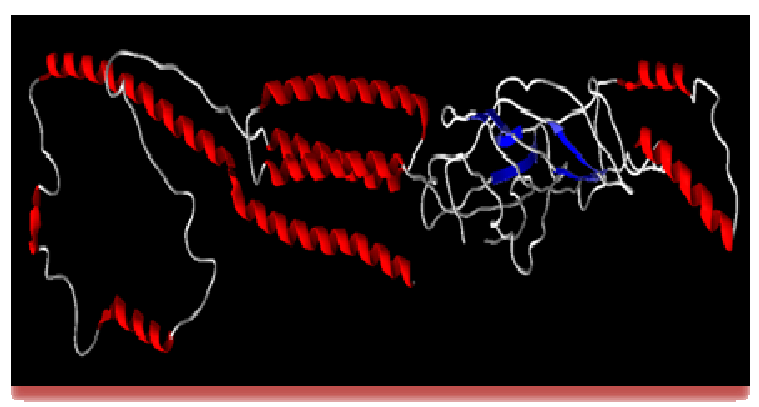

Figure 2. Backbone structure of 5-hydroxytryptamine $1_{\mathrm{A}}$ receptor.

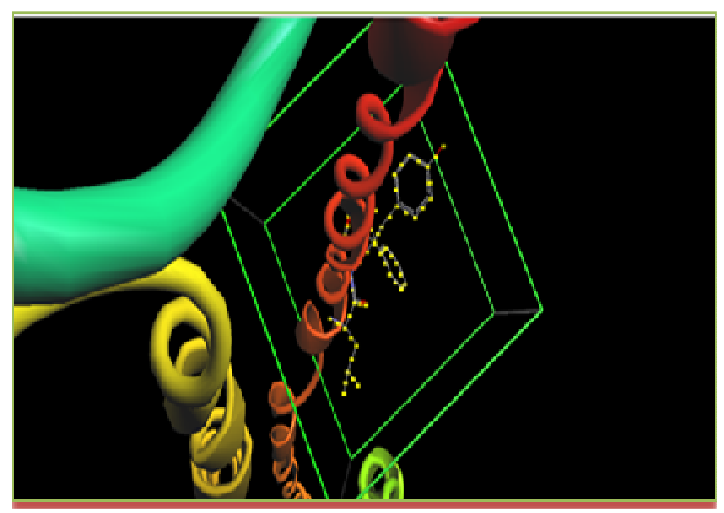

Figure 3. Active binding site of $5 \mathrm{HT}_{1 \mathrm{~A}}$ protein. the quadrangle. The red regions in the graph indicate the most allowed regions whereas the yellow regions represent allowed regions. In this protein model, $74.3 \%$ of the residues were in the most favored region, $21.3 \%$ in allowed region, $4.5 \%$ in generously allowed region and $0 \%$ of the residues lying in the disallowed regions.

\section{Binding site of the protein}

The detection of ligand-binding sites is often the starting point for protein function identification and drug discovery. 3D ligand site server predicted active site of the protein $5 \mathrm{HT}_{1 \mathrm{~A}}$ (Figure 3 ) with a higher average precision. The active site of $5 \mathrm{HT}_{1 \mathrm{~A}}$ comprises of amino acid residues such as LEU 453, PHE 454, and TYR 457.

\section{Docking}

To understand the interactions between the ligands and $5 \mathrm{HT} 1 \mathrm{~A}$ protein and to explore their binding mode, docking study was performed using Argus dock available under ArgusLab 4.0.1. The ligands were created and prepared for the docking procedure using ChemSketch and ArgusLab. The structures of the ligands obtained are shown in Figure 4. All the molecules satisfied the Lipinski's drug properties (Mathew et al., 2010) (Table 1). 
Table 1. Lipinski properties of the compounds.

\begin{tabular}{lccccc}
\hline Molecule & Molecular weight & Log P & H-Donor & H-Acceptor & Number of rotatable bonds \\
\hline Gingerol & 294.391 & 3.217 & 2 & 4 & 10 \\
Shogoal & 276.376 & 4.348 & 1 & 3 & 9 \\
Imipramine & 280.415 & 4.161 & 0 & 2 & 4 \\
\hline
\end{tabular}

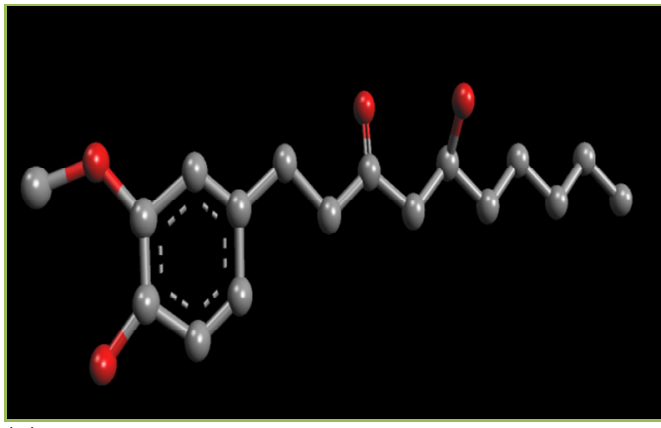

(a)

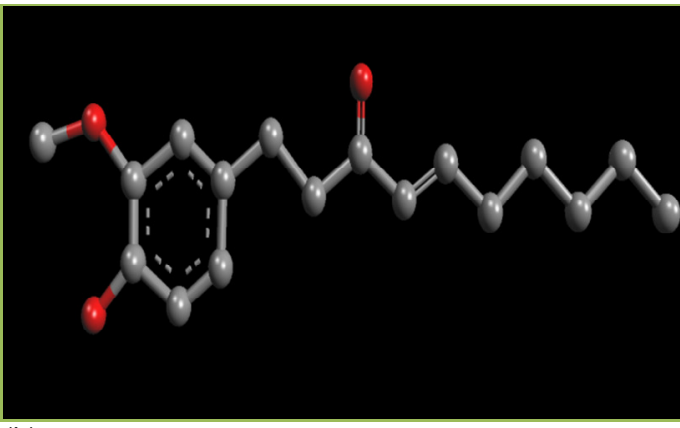

(b)

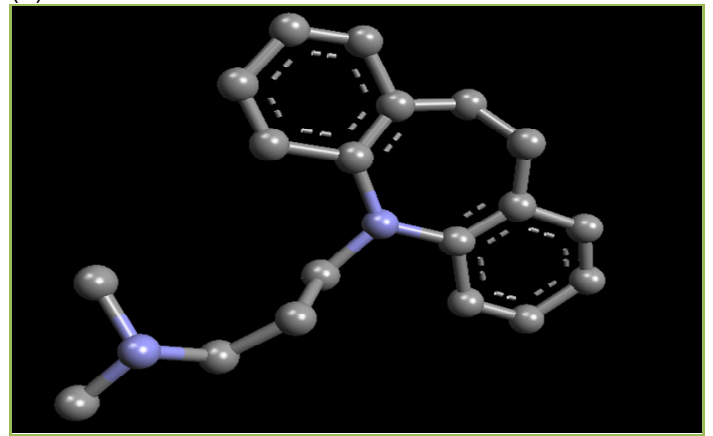

(c)

Figure 4. Structure of ligands. (a) Gingerol; (b) shogoal; (c) imipramine.

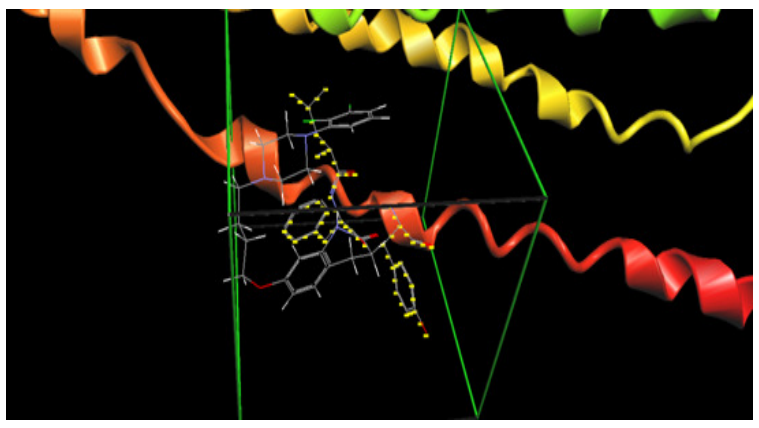

Figure 5. 5 $\mathrm{HT}_{1 \mathrm{~A}}$-gingerol complex: Yellow color represents binding site residues; grey color represents the ligand; blue, red and green color represents the rest of the protein. Binding mode of Shogoal with $5 \mathrm{HT}_{1 \mathrm{~A}}$ protein.

\section{Docking scores}

The docking scores were highest for shogoal with -9.0267



Figure 6. $5 \mathrm{HT}_{1 \mathrm{~A}}$-shogoal complex: Yellow color represents binding site residues; grey color represents the ligand; blue, red and green color represents the rest of the protein

$\mathrm{Kcal} / \mathrm{mol}$, gingerol with $-8.41044 \mathrm{Kcal} / \mathrm{mol}$ and imipramine with $-8.21131 \mathrm{Kcal} / \mathrm{mol}$, respectively and the binding mode of ligands with $5 \mathrm{HT}_{1 \mathrm{~A}}$ is shown in Figures 5 to 8. 


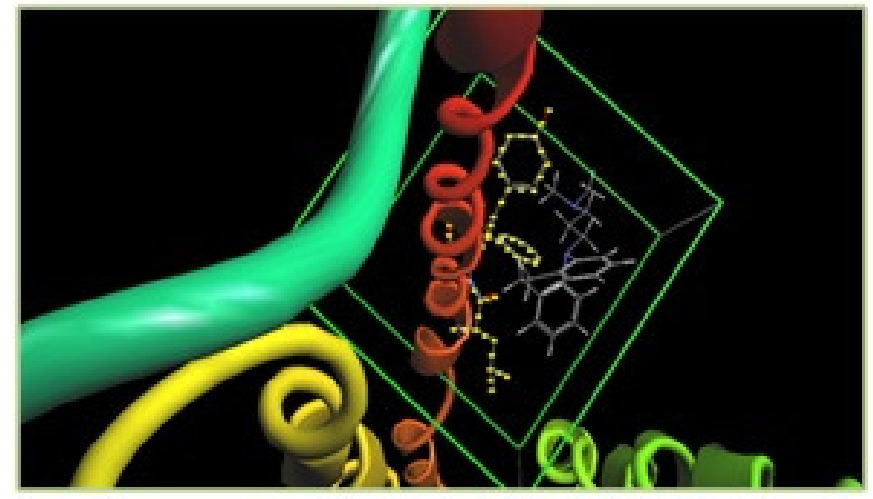

Figure 7. Binding mode of imipramine with $5 \mathrm{HT}_{1 \mathrm{~A}}$ protein.

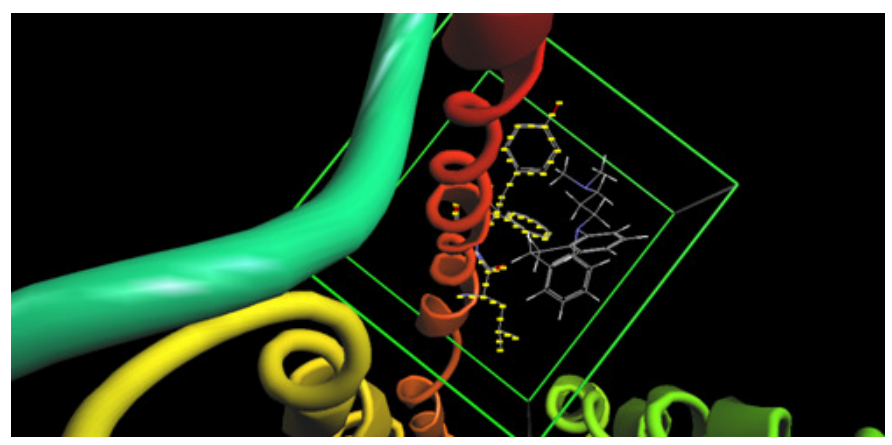

Figure 8. $5 \mathrm{HT}_{1 \mathrm{~A}}$-imipramine complex: Yellow color represents binding site residues; grey color represents the ligand; blue, red and green color represents the rest of the protein.

\section{CONCLUSION}

The current therapeutic goal in the treatment of major depression is to improve quality of life by normalizing mood and reversal of functional and social disabilities associated with depression. Molecular docking continues to hold great promise in the field of computer based drug design which screens small molecules by orienting and scoring them in the binding site of a protein. Gingerol and shogoal interacted with LEU 453, PHE 454, and TYR 457 with high affinity. Comparative docking analysis of gingerol and shogoal with commonly used drug for treatment of depression such as imipramine also suggests that these compounds can be an alternative source for treatment of depression. Therefore, this approach is valuable for drug discovery process and therapy of depression. Hence, now there is a need to study the pharmacological activity of these compounds in in vivo models.

\section{REFERENCES}

Jones TD (1999). GenThreader: An efficient and reliable protein folds recognition method for genomic sequence. J. Mol. Biol. 287:797-815.

Mathew B, Vakketh SS, Kumar SS (2010). Synthesis, molecular properties and anthelmintic activity of some schiff bases of 1, 3, 4 thiadiazole derivatives. Scholars Research Library. Der Pharma Chemica. 2(5):337-43.

Nievergelt A, Huonker P, Schoop R, Altmann KH, Gertsch J (2010). Identification of serotonin $5-\mathrm{HT} 1 \mathrm{~A}$ receptor partial agonists in ginger. Bioorg. Med. Chem. 18:3345-51.

Rajput MS, Sinha S, Mathur V, Agrawal P (2011). Herbal Antidepressants. IJPFR.1 (1):159-69.

Vaseeharan B, Valli SJ (2011). Insilico homology modeling of prophenoloxidase activating factor serine proteinase gene from the haemocytes of Fenneropenaeus indicus. J. Proteomics Bioinform. 4:53-57.

Zhang Y (2008). I-TASSER server for protein 3D structure prediction. BMC Bioinformatics 9:40. 